\title{
Avaliação Psicológica na pós-graduação: um panorama das regiões Centro-Oeste, Nordeste e Norte do Brasil
}

Gabriel Vitor Acioly Gomes . Universidade São Francisco

Camila Costa e Silva. Pontifícia Universidade Católica de Goiás

Daniela Sacramento Zanini. Universidade Católica de Goiás

Lucila Moraes Cardoso. Universidade Estadual do Ceará

\section{Resumo}

A Pós-graduação Stricto Sensu em Psicologia tem se desenvolvido no Brasil com assimetrias regionais. Objetivou-se no presente estudo realizar uma caracterização atual sobre os dados de distribuição do ensino de Avaliação Psicológica no âmbito da Pós-graduação Stricto Sensu nas regiões Centro-Oeste, Nordeste e Norte do Brasil. O material de pesquisa foi coletado no Centro de Gestão e Estudos Estratégicos, Plataforma Lattes, Plataforma Sucupira e no site do Instituto Brasileiro de Avaliação Psicológica (IBAP). Os resultados evidenciaram um aumento na quantidade de pós-graduados, em Programas de Pós-graduação Stricto Sensu e nos laboratórios de pesquisa. O perfil da Pós-graduação Stricto Sensu em Avaliação Psicológica é de linhas de pesquisa em programas amplos da Psicologia e não em programas específicos de avaliação psicológica. $O$ aumento observado foi insuficiente para superar as assimetrias entre regiões na quantidade de Programas de Pós-graduação Stricto Sensu, o que reforça a necessidade de estratégias para fortalecê-los nas regiões Centro-Oeste, Nordeste e Norte do Brasil.

Palavras-chave: avaliação psicológica; pós-graduação; formação do psicólogo.

\section{Abstract}

Psychological assessment on graduate: an overview of the Central-west, Northeast and North regions of Brazil. The Stricto Sensu Postgraduate Education in Psychology has developed in Brazil with regional asymmetries. The objective of this study was to carry out a current characterization of the distribution data of the Psychological Assessment education in the Stricto Sensu Graduate Program in the Central-west, Northeast and North regions of Brazil. The research material was collected at the Centro de Gestão e Estudos Estratégicos, Plataforma Lattes, Plataforma Sucupira and on the website of the Brazilian Institute of Psychological Assessment (IBAP). The results evidenced an increase in the number of postgraduates, in Stricto Sensu Graduate Programs and in the research laboratories. The Profile of Stricto Sensu Postgraduate in Psychological Assessment is from lines of research in broad Psychology programs rather than specific psychological assessment programs. The observed increase was insufficient to overcome the asymmetries between regions in the number of Stricto Sensu Postgraduate Programs, which reinforces the need for strategies to strengthen them in the Central-west, Northeast and North regions of Brazil.

Keywords: psychological assessment; postgraduated training; psychologist education.

\section{Resumen}

Evaluación Psicológica en el postgrado: una visión general de las regiones Centro-Oeste, Nordeste y Norte de Brasil. El Postgrado Stricto Sensu en Psicología se ha desarrollado en Brasil con asimetrías regionales. Se objetivó en el presente estudio realizar una caracterización actual sobre los datos de distribución de la enseñanza de Evaluación Psicológica en el ámbito del Postgrado Stricto Sensu en las regiones Centro-Oeste, Nordeste y Norte de Brasil. El material de investigación fue recolectado en el Centro de Gestão e Estudos Estratégicos, Plataforma Lattes, Plataforma Sucupira y en el sitio del Instituto Brasileño de Evaluación Psicológica (IBAP). Los resultados evidenciaron un aumento en la cantidad de postgrados, en Programas de Postgrado Stricto Sensu y en los laboratorios de investigación. El perfil de Postgrado Stricto Sensu en evaluación psicológica proviene de líneas de investigación en programas amplios de Psicología en lugar de programas específicos de evaluación psicológica. El aumento observado fue insuficiente para superar las asimetrías entre regiones las regiones en el número de Programas de Posgrado Stricto Sensu, lo que refuerza la necesidad de estrategias para fortalecerlos en las regiones Centro-Oeste, Nordeste y Norte de Brasil.

Palabras clave: evaluación psicológica; posgrado; formación del psicólogo. 
A Pós-graduação Stricto Sensu brasileira é uma iniciativa recente e passa por um contínuo refinamento, visando o aprimoramento da natureza acadêmica e das pesquisas de cunho científico (Tourinho \& Bastos, 2010). Foi em 1965 que a Coordenação de Aperfeiçoamento de Pessoal de Nível Superior (CAPES) elencou a Pós-graduação Stricto Sensu como importante estratégia de mudança na educação brasileira. Posteriormente, na década de 1970, a CAPES foi vinculada ao Ministério da Educação e se tornou responsável pela organização, gerenciamento e avaliação da Pós-graduação Stricto Sensu brasileira (Hutz, Rocha, Spink, \& Menandro, 2010; Yamamoto \& Menandro, 2004).

A Pós-graduação Stricto Sensu no Brasil, desde então, tem se modificado e crescido em termos de quantidade de programas, de alunos, professores, bolsas, entre outros indicadores (Centro de Gestão e Estudos Estratégicos [CGEE], 2016; Cirani, Campanario, \& Silva, 2015; CAPES, 2019; Instituto Nacional de Estudos e Pesquisas Educacionais Anísio Teixeira [INEP], 2016). Apesar disso se observa uma assimetria regional desses programas (Tourinho \& Bastos, 2010). Ao considerar apenas a distribuição dos Programas de Pós-graduação Stricto Sensu em 2017, de acordo com os dados do Sistema de Informações Georreferenciadas, do total de 4.296 programas de todas as áreas de conhecimento, 66,13\% estão localizados nas regiões Sul e Sudeste do Brasil (CAPES, 2019). Por conseguinte, a quantidade de pessoas tituladas na pós-graduação, entre o período de 2004 a 2014, é predominante nas regiões Sudeste e Sul quando comparadas às demais regiões brasileiras (INEP, 2016).

De acordo com o Centro de Gestão e Estudos Estratégicos (2016), existem 704.337 pós-graduados em todas as áreas de conhecimento no Brasil, sendo aproximadamente $26,60 \%$ doutores e $73,40 \%$ mestres. Contudo, quando se analisa o número de mestres e doutores que se cadastraram na Plataforma Lattes este número decresce para um total de 215.449 , sendo $61,56 \%$ doutores e $38,44 \%$ mestres. De forma mais específica, observa-se que 132.630 doutores $(70,79 \%)$ e 82.819 mestres $(16,01 \%)$ brasileiros se cadastraram na Plataforma Lattes. Essa diferença entre a quantidade de pessoas tituladas e o número de pessoas cadastradas pode ter a ver com a natureza acadêmica da Plataforma Lattes.

No sentido de ampliar o acesso à Pós-graduação Stricto Sensu no Brasil, a CAPES implementou políticas de indução para o seu financiamento, com investimento de recursos de custeio e de capital para pesquisas e formação de pessoas, bem como com o aumento de programas e bolsas. Exemplos de programas para ampliar o acesso à Pós-graduação, tendo a ênfase nas regiões Centro-Oeste, Nordeste e Norte, são o Acelera Amazônia, para aumentar a quantidade de pesquisadores e grupos de pesquisa na região amazônica; o DINTER Novas Fronteiras, para propiciar a formação docente em nível de doutorado nas instituições federais e estaduais; o PRODOUTORAL, para estimular o planejamento de formação docente nos Institutos Federais; e o PROCAD Novas Fronteiras, para consolidar os novos Programas de Pós-graduação Stricto Sensu que geralmente têm nota 3 (CAPES, 2010).

Em termos da estruturação dos programas de pós-graduação, estes são formados por linhas de pesquisas que concentram os grupos de pesquisas cadastrados no CNPq e laboratórios. A linha de pesquisa de um programa indica os temas de estudos científicos com base na tradição investigativa dos quais se originam os projetos de pesquisas a serem executados (CNPq, 2019b). O grupo de pesquisa, por outro lado, se caracteriza por um conjunto de pesquisadores e estudantes organizados em torno de uma liderança com experiência e competência técnico-científica (CNPq, 2019a), sem necessariamente estar atrelado a um Programa de Pós-graduação. Por fim, os laboratórios de pesquisa são importantes espaços de fomento de pesquisa, em especial, pela integração entre a Pós-graduação Stricto Sensu e a graduação, com a participação de docentes, pós-graduandos e bolsistas de iniciação científica.

Os laboratórios podem existir nas instituições de ensino sem que haja um registro nos órgãos de fomento à pesquisa ou vínculo com um Programa de Pós-graduação. Ou seja, são espaços que favorecem a troca de experiências entre pesquisadores sendo necessário para sua criação um nível de maturidade e consolidação das pesquisas dentro das instituições (Bastos et al., 2015). Assim, é importante que se busque uma relação de diálogo e construção entre a graduação e a Pós-graduação Stricto Sensu, evitando um distanciamento e permitindo um crescimento conjunto (Menandro, Tourinho, Bastos, \& Yamamoto, 2013; Yamamoto, 2006).

No que se refere especificamente à Pós-graduação Stricto Sensu em Psicologia o panorama não é diferente do geral, ou seja, também existe uma assimetria entre a distribuição dos programas nas regiões brasileiras (Bastos, Tomanari, Trindade, \& Andery, 2015; Féres-Carneiro, 
Bastos, Feitosa, Seidl-de-Moura, \& Yamamoto, 2010; Tourinho \& Bastos, 2010). Logo, as políticas de indução também contribuem com a tentativa de reduzir as assimetrias dos Programas de Pós-graduação Stricto Sensu em Psicologia.

Outra entidade que busca congregar pesquisadores vinculados a diferentes Programas de Pós-graduação Stricto Sensu em Psicologia no Brasil é a Associação Nacional de Pesquisa e Pós-Graduação em Psicologia (ANPEPP). A ANPEPP foi fundada em 1983 com o objetivo de unir Programas de Pós-graduação Stricto Sensu de instituições de ensino superior para fomentar e estimular a formação de profissionais para pesquisa e Pós-graduação Stricto Sensu em Psicologia, valorizando perspectivas de integração entre os programas em todo o Brasil (ANPEPP, 2019). Atualmente, se reconhecem suas amplas contribuições em prol do desenvolvimento da Pós-graduação Stricto Sensu em Psicologia no Brasil. Entre elas destaca-se o fomento de discussões de políticas científicas, políticas de internacionalização, incentivo ao intercâmbio entre pesquisadores e produção intelectual (Gomes \& Fradkin, 2015; Guzzo, Linhares, Teodoro, \& Koller, 2015).

A CAPES e CNPq organizam os programas de acordo com as grandes áreas, mas também de acordo com as áreas de concentração. A área de concentração diz respeito aos interesses investigativos do grupo. Dentre as diversas áreas de concentração existentes nos Programas de Pós-graduação Stricto Sensu em Psicologia, destaca-se a de Fundamentos e medidas em Psicologia, tema de interesse da presente pesquisa. Essa área tem um caráter técnico-científico e é reconhecida por possibilitar a avaliação do funcionamento psicológico de um sujeito por meio do uso de diversos recursos de medidas e de investigações (Bardagi, Teixeira, Seganinazi, Schelini, \& Nascimento, 2015; Borsa, 2016). Assim, ao se investigar de maneira específica a área de Fundamentos e medidas em Psicologia, na qual estão inseridas as pesquisas em Avaliação Psicológica, o que se evidencia é um cenário semelhante de assimetrias regionais. O primeiro Programa de Pós-graduação Stricto Sensu em Psicologia com concentração em Avaliação Psicológica do Brasil é o da Universidade São Francisco (USF), localizado na região Sudeste (Gouveia, 2018; Primi, 2010).

Considerando-se a amplitude de áreas da Psicologia e as especificidades da Avaliação Psicológica, de fato, não é esperado que haja diversos Programas de Pós-graduação Stricto Sensu com concentração nessa área. Pesquisadores têm apontado pouca ênfase curricular na área de Avaliação Psicológica nos cursos de Psicologia (Noronha, 2003), outros apontam a necessidade de diretrizes e um conjunto de disciplinas a serem ofertadas (Nunes et al., 2012). Os professores que ministram disciplina nessa área costumam não ter uma identidade com ela, durante o ensino é dado um foco na testagem psicológica e são usados materiais desatualizados em aula, entre outros aspectos que tendem a propiciar uma formação inadequada (Ambiel, Zuanazzi, Sette, Costa, \& Cunha, 2019; Bardagi et al., 2015; Borsa, 2016; Freires, Silva Filho, Monteiro, Loureto, \& Gouveia, 2017; Noronha, 2003). Desta forma, a falta de uma boa formação na Pós-graduação Stricto Sensu pode acarretar professores menos aptos a atuarem na graduação. Além disso, o caminho traçado na formação inicial pode dificultar o interesse e a aproximação de alunos por um aprofundamento na Pós-graduação Stricto Sensu em Avaliação Psicológica.

Essa aproximação entre pesquisadores de diferentes níveis de ensino e regiões do Brasil costuma ocorrer nos congressos científicos. No caso da área de Avaliação Psicológica, destacam-se os congressos organizados pelo Instituto Brasileiro de Avaliação Psicológica (IBAP) e pela Associação Brasileira de Rorschach e Métodos Projetivos (ASBRo). Essas têm o objetivo de promover o desenvolvimento da área de Avaliação Psicológica, seja no âmbito da pesquisa, ensino ou atuação do profissional de Psicologia, bem como defender e propor medidas de apoio e incentivo à Avaliação Psicológica e às atividades relacionadas (IBAP, 2015), tratando-se de instituições reconhecidas na área (Gouveia, 2018; Primi, 2010).

Tendo em vista as demandas para qualificação dos profissionais para atuarem na área de Avaliação Psicológica, bem como da adequada formação de docentes e pesquisadores, o objetivo do presente estudo foi realizar uma caracterização atual sobre os dados de distribuição do ensino de Avaliação Psicológica no âmbito da Pós-graduação Stricto Sensu nas regiões Centro-Oeste, Nordeste e Norte do Brasil. De maneira específica, no presente artigo buscou-se: a) comparar o número de profissionais com pós-graduação, em todas as áreas de conhecimento, por região e por estado do Brasil; b) distinguir a quantidade de pós-graduados em Psicologia por região brasileira; c) identificar os Programas de Pós-graduação Stricto Sensu em Psicologia com linha de pesquisa em Avaliação Psicológica por regiões do Brasil e d) verificar a distribuição dos 
laboratórios de pesquisa desta área de estudo. Espera-se, a partir dos dados levantados, discutir sobre o panorama atual e os desafios a serem percorridos para o desenvolvimento dessa área.

\section{Método}

\section{Composição do Corpus de Pesquisa}

Foi pesquisada a quantidade de títulos concedidos e o número de doutores e mestres em todas as áreas de conhecimento no cenário brasileiro. Para isso utilizou-se o estudo da demografia da base técnico-científica brasileira (CGEE, 2016). Contudo, este estudo não apresenta a distribuição de mestres e doutores por áreas de conhecimento nas regiões do Brasil.

Repetiu-se a busca, desta vez limitando para a área de conhecimento da Psicologia, na Plataforma Lattes do Conselho Nacional de Desenvolvimento Científico e Tecnológico (CNPq), para ter acesso ao número de doutores e mestres em Psicologia, na área de ensino e pesquisa. A busca seguiu os caminhos de entrada no site http://lattes.cnpq.br/ e em seguida no item "Distribuição Geográfica". Para os filtros na distribuição geográfica seleciona-se em base o item "Doutores" e, posteriormente, "Mestres". Em tipo de atividade opta-se por "Ensino e Pesquisa" e em "totais" escolhe-se o "Número para 100 mil habitantes".

No que diz respeito às informações dos cursos de psicologia avaliados pela Coordenação de Aperfeiçoamento de Pessoal de Nível Superior (CAPES), realizou-se a estratégia de consulta no site https://sucupira.capes.gov.br/sucupira/. Seguiu uma sequência de caminhos, a saber, "Cursos Avaliados e Reconhecidos", "Por Área de Avaliação" e "Psicologia". Das instituições cadastradas, selecionaram-se as pertencentes das regiões Centro-Oeste, Nordeste e Norte e foram averiguadas informações sobre existência de área de concentração ou linha de pesquisa em Avaliação Psicológica, nível e nota do curso. Em seguida, foram acessados os sites das universidades para a conferência e quantificação das linhas de pesquisa em Avaliação Psicológica nos Programas de Pós-graduação Stricto Sensu.

Por fim, foi investigada a quantidade e as respectivas localizações dos laboratórios de pesquisa em Avaliação Psicológica no Brasil, conforme listagem obtida no site do IBAP. Essas informações estão em processo de atualização e, portanto, a listagem obtida é de maio de 2015.

\section{Resultados}

A Figura 1 apresenta o número e percentagem de títulos de doutorado e mestrado concedidos por região no Brasil em 2014 segundo o Estudo da demografia da base técnico-científica brasileira (CGEE, 2016).

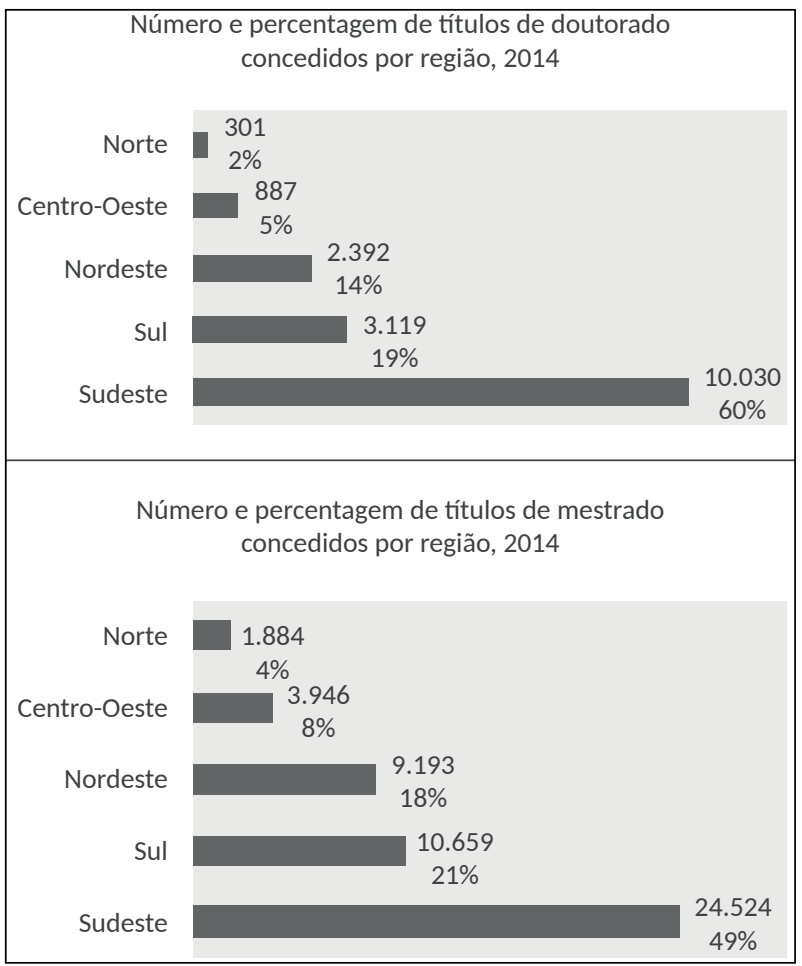

Figura 1. Distribuição de Títulos de Doutorado e Mestrado Concedidos por Região no Brasil.

Fonte: Dados recuperados da CGEE (2016).

Conforme observa-se na Figura 1, de acordo com o Centro de Gestão e Estudos Estratégicos (2016) foram concedidos no Brasil 16.729 títulos de doutorado em 2014, sendo a maioria no Sudeste, representando $60 \%$, com 10.030 títulos, seguido do Sul com 3.119 títulos (19\%), Nordeste com 2.392 títulos (14\%), CentroOeste com 887 títulos (5\%) e, por último, o Norte com 301 títulos (2\%). No âmbito do mestrado, foram concedidos 50.206 títulos em 2014, destes $49 \%$ concentram-se no Sudeste com 24.524 títulos, posteriormente, vem o Sul com 10.659 títulos (21\%), Nordeste com 9.193 títulos (18\%), Centro-Oeste com 3.946 títulos (8\%) e o Norte com 1.884 títulos concedidos (4\%). Contudo, esses dados foram avaliados em termos de números brutos. Para avaliar em termos de taxa populacional a Figura 2 apresenta a distribuição por 100 mil habitantes dos títulos de doutorado e mestrado por unidade de federação em 2014 (CGEE, 2016). 


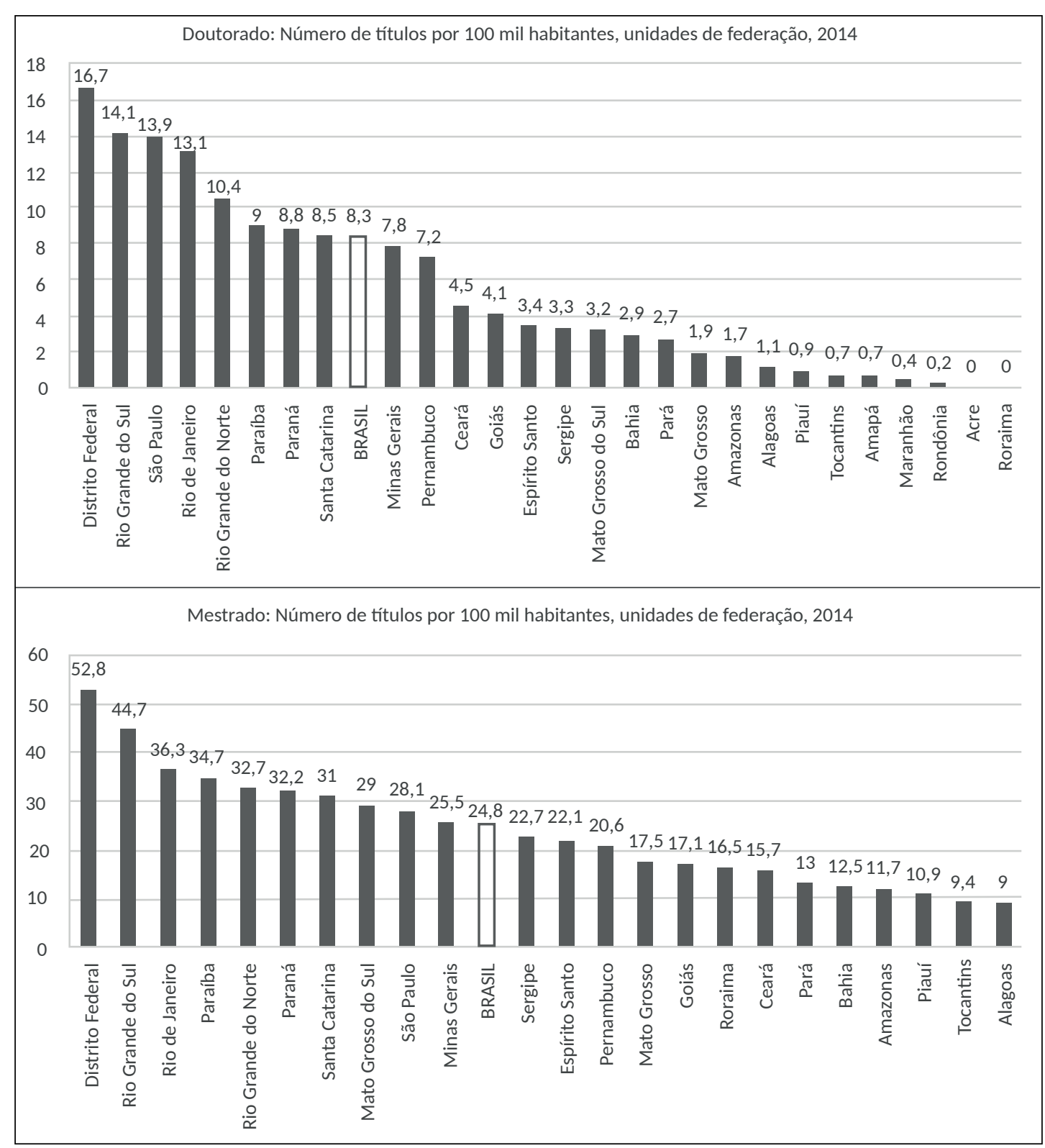

Figura 2. Distribuição de Títulos Concedidos de Doutorado e Mestrado por 100 Mil Habitantes por Estado no Brasil. Os valores que aparecem no gráfico são referentes ao ano de 2014. Foram suprimidos os dados referentes aos títulos de mestres quando a proporção de títulos concedidos na UF era menor que $0,2 \%$

Fonte: Dados obtidos da CGEE (2016).

De acordo com a Figura 2, o Brasil apresenta um número de 8,3 doutores por 100 mil habitantes. Os estados que estão acima deste número são o Distrito Federal com 16,7, o Rio Grande do Sul com 14,1, São Paulo com 13,9, Rio de Janeiro 13,1, Rio Grande do Norte com 10,4, Paraíba com 9, Paraná com 8,8 e Santa Catarina com 8,5 doutores. Em relação aos mestres, o Brasil apresenta 24,8 mestres por 100 mil habitantes. Os estados que apresentam índices acima deste são o Distrito Federal com 52,8, o Rio Grande do Sul com 44,7, Rio de Janeiro com 36,3, Paraíba com 34,7, Rio Grande do Norte com 32,7, Paraná com 32,2, Santa Catarina com 31, Mato Grosso do Sul com 29, São Paulo com 28,1 e Minas Gerais com 
25,5. Considerando os doutores, o Pará com 2,7 e o Amazonas com 1,7, apresentam os maiores índices da região Norte, os demais são inferiores à um doutor por 100 mil habitantes. Em relação aos mestres, Roraima com 16,5, Pará com 13 e Amazonas com 11,7 mestres por 100 mil habitantes apresentam os maiores números da região Norte, os demais apresentam índices inferiores a 10 mestres.

Observa-se que a taxa de distribuição de doutores e mestres nas unidades de federação é diferente da concessão destes títulos. Conforme Figura 2, o Distrito Federal está à frente dos títulos de doutores e mestres, consequentemente, é o responsável por elevar os índices do Centro-Oeste visto que nenhum outro estado da região está acima da média do Brasil de 8,3 emissão de títulos de doutorado. No que se refere aos títulos de mestres, Mato Grosso do Sul foi o único outro estado da região Centro-Oeste que superou a média brasileira. Na região Nordeste dois estados elevaram os índices de forma semelhante ao Distrito Federal, tanto em doutores quanto mestres, a Paraíba e o Rio Grande do Norte estão acima da média do Brasil. Por fim, todos os estados da região Norte ficaram abaixo da média brasileira. À exceção do Espírito Santo (ambas titulações) e Minas Gerais (títulos de doutores), todos os estados da região Sul e Sudeste estavam acima da média brasileira.

Para avaliar o número de mestres e doutores especificamente na área de Psicologia recorreu-se a busca na Plataforma Lattes. Verificou-se que o número de pós-graduados dessa área cadastrados na plataforma corresponde, respectivamente, a $4,41 \%$ e $13,41 \%$ dos doutores e mestres. A Figura 3 apresenta a distribuição por 100 mil habitantes dos doutores e mestres em psicologia cadastrados na Plataforma Lattes.

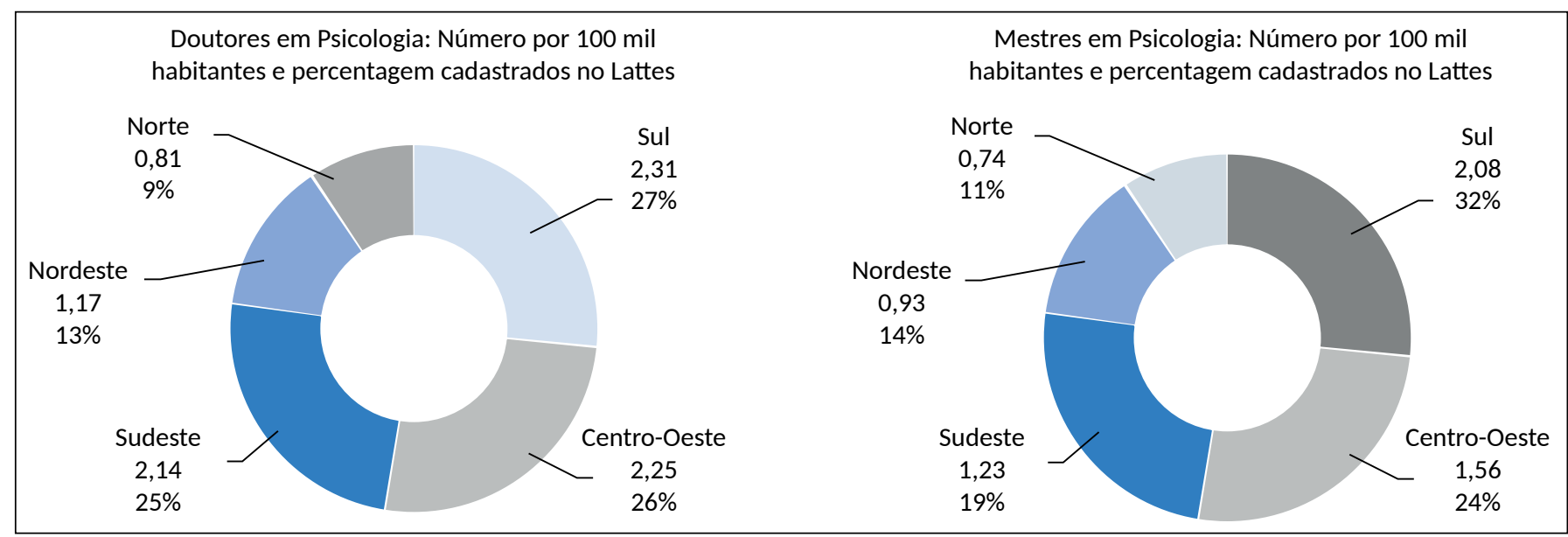

Figura 3. Quantidade de Doutores e Mestres em Psicologia Cadastrados na Plataforma Lattes por 100 Mil Habitantes por Região no Brasil. Fonte: Dados recuperados na Plataforma Lattes (2019).

Ao considerar a distribuição por 100 mil habitantes de doutores por região, de acordo com a Figura 3, a região Sul apresentou o maior número, com 2,31 doutores, em seguida a região Centro-Oeste com 2,25, região Sudeste com 2,14, região Nordeste com 1,17 e, por último, a região Norte com 0,81. A distribuição de mestres pelas regiões no Brasil seguiu proporções semelhantes à de doutores. Assim, o Sul apresentou 2,08 mestres por 100 mil habitantes, seguido pelo Centro-Oeste com 1,56 mestres, Sudeste com 1,23, Nordeste com 0,93 e, por último, o Norte com 0,74 mestres.
$\mathrm{Na}$ lista geral de cursos avaliados e reconhecidos foram apresentados 100 Programas de Pós-graduação Stricto Sensu distribuídos em 72 Instituições de Ensino Superior (IES), das quais 29 instituições estavam localizadas fora do eixo Sul-Sudeste, representando 40,27\% do total. Na Tabela 1 constam os dados acessados no site da Plataforma Sucupira sobre os Programas de Pós-graduação Stricto Sensu em Psicologia fora do eixo Sul-Sudeste que possuem cursos avaliados e reconhecidos pela CAPES. As informações relativas à linha de pesquisa ou área de concentração no campo de Avaliação Psicológica foram pesquisadas nos sites das IES na área designada à Pós-graduação Stricto Sensu. 
Tabela 1. Programas de Pós-graduação em Psicologia e Notas do Curso

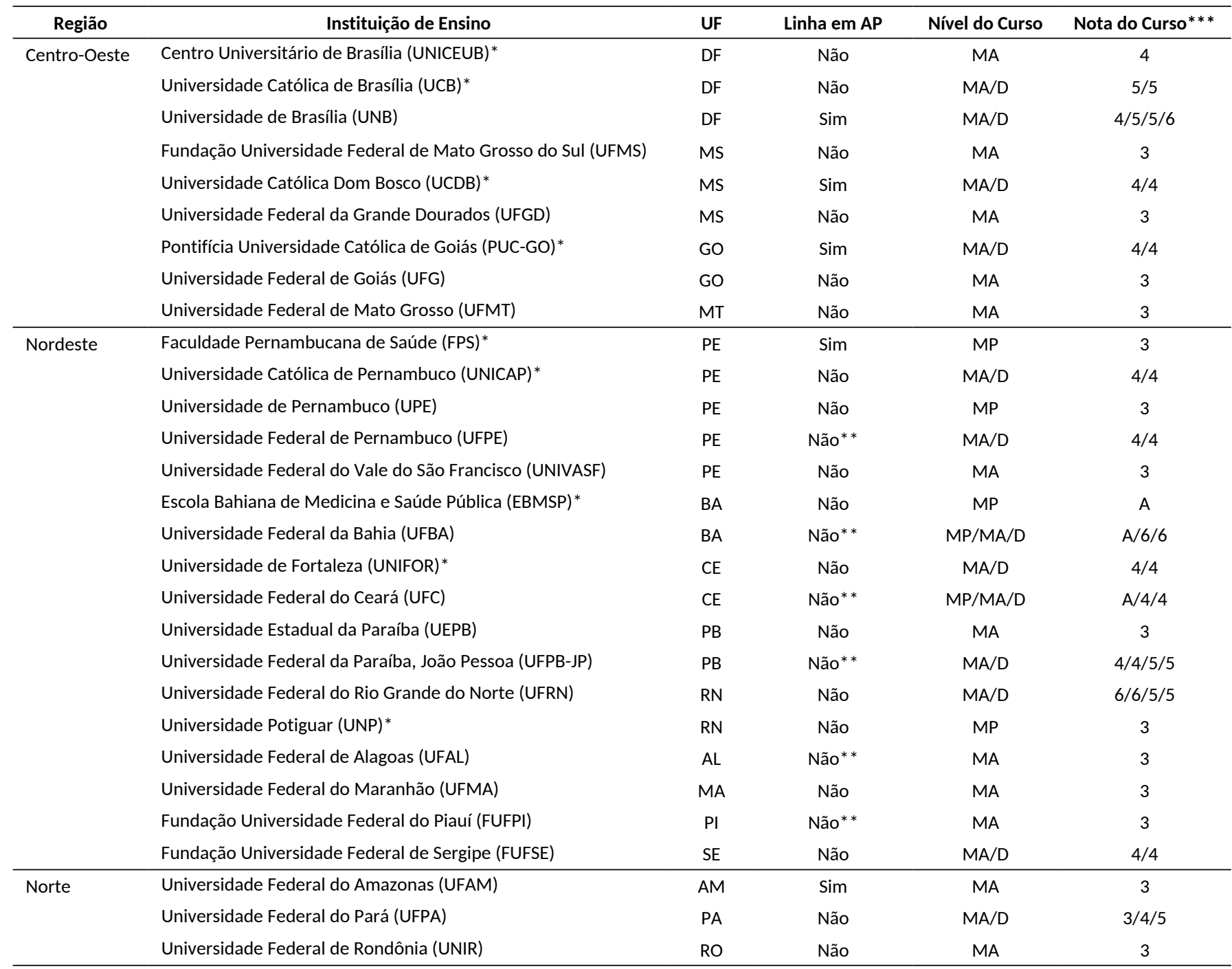

Nota. MA - Mestrado Acadêmico, MP - Mestrado Profissional e D - Doutorado.

*Instituições particulares. **Embora não tenham linha específica na área de Avaliação Psicológica, há grupos de pesquisa registrados no Diretório de grupos de Pesquisa do CNPq. ***Algumas instituições oferecem mais de um Programa de pós-graduação e cada programa recebe uma nota CAPES específica. Na Tabela estão as notas correspondentes, conforme Plataforma Sucupira.

Fonte: Dados recuperados da Plataforma Sucupira.

Conforme a Tabela 1, das regiões conferidas, destaca-se a existência de Instituições de Ensino Superior (IES) com Programas de Pós-graduação Stricto Sensu em Psicologia em Pernambuco (PE) $(n=5)$, no Distrito Federal (DF) $(n=3)$ e no estado de Mato Grosso do Sul (MS) $(n=3)$. Algumas destas IES apresentam mais de um Programa de Pós-graduação Stricto Sensu em Psicologia. Outros cinco estados apareceram com duas IES com Programas de Pós-graduação Stricto Sensu em Psicologia, sendo esses, Bahia (BA), Ceará (CE), Goiás (GO), Paraíba (PB) e Rio Grande do Norte (RN). Por fim, oito estados apresentaram uma IES com Programa de Pós-graduação Stricto Sensu em Psicologia listado na
Plataforma Sucupira, a saber, o estado de Alagoas (AL), Amazonas (AM), Maranhão (MA), Mato Grosso (MT), Pará (PA), Piauí (PI), Rondônia (RO) e Sergipe (SE).

Nota-se, ainda na Tabela 1, que cinco IES possuíam Programas de Pós-graduação Stricto Sensu com linha de pesquisa em Avaliação Psicológica. São elas: Avaliação e Medidas em Psicologia (UnB), Avaliação Psicológica e Promoção de Ações em Saúde (FPS-PE), Saúde: processos, avaliação e intervenção (PUC-GO), Avaliação e assistência em saúde (UCDB-MS) e Processos Psicológicos e Saúde (UFAM-AM).

Constatou-se também que seis IES não tinham linha específica em Avaliação Psicológica, mas 
contemplavam grupos de pesquisa no diretório de pesquisa do CNPq (Tabela 1). Os grupos de pesquisa que constam no diretório de pesquisa do CNPQ são o Núcleo de Estudos e Pesquisas em Avaliação Psicológica (NEPAP) da UFPB-JP, o Laboratório Cearense de Psicometria (LACEP) da UFC-CE, o Núcleo de Estudos em Avaliação Psicológica (NEAP) da UFPE-PE, o Laboratório de Psicometria e Avaliação Psicológica da UFAL-AL, o Núcleo de Instrumentos e Medidas (NIM) da UFBA-BA e o Laboratório de Avaliação Psicológica do Delta (LABAP-D) da UFPI-PI.
Realizou-se uma busca no site do IBAP para a coleta dos dados referentes aos laboratórios de pesquisa em Avaliação Psicológica distribuídos por região no Brasil. Essas informações estavam expostas em um catálogo e constatou-se a existência de 45 laboratórios. Observa-se que, do total de laboratórios, o Sudeste apresentou 20 (44\%), o Sul 12 (27\%), Nordeste nove (20\%), Centro-Oeste dois $(4,5 \%)$ e Norte também dois $(4,5 \%)$. Tendo como ênfase as regiões fora do eixo Sul-Sudeste, foram apresentados na Tabela 2 os nomes dos laboratórios em função da região, suas respectivas IES e estados de origem.

Tabela 2. Relação de Laboratórios de Avaliação Psicológica Fora do Eixo Sul-Sudeste

\begin{tabular}{llll}
\hline \multicolumn{1}{c}{ Região } & \multicolumn{1}{c}{ Instituição de Ensino } & UF & \\
\hline Centro-Oeste & Universidade de Brasília & DF & Laboratório de Pesquisa em Avaliação e Medida - LABPAM \\
& Universidade de Brasília & DF & Laboratório de Saúde Mental e Cultura - LSMC \\
\hline Nordeste & Universidade Federal do Ceará & CE & Laboratório Cearense de Psicometria - LACEP \\
& Universidade Estadual do Ceará & CE & Laboratório de Estudos em Avaliação Psicológica - LEAPSI \\
& Universidade Federal da Paraíba & PB & Bases Normativas do Comportamento Social - BNCS \\
& Universidade Federal da Paraíba & PB & Grupo de Pesquisa em Avaliação e Medidas Psicológicas - GPAMP \\
& Universidade do Piauí* & PI & Núcleo de Avaliação Psicológica do Delta - NAPSID \\
& Universidade Federal do Piauí & PI & Laboratório de Avaliação Psicológica do Delta - LABAP-D \\
& Universidade Federal de Alagoas & AL & Laboratório de Avaliação e Medida Cognitivo Emocional - LAMCE \\
& Universidade Federal da Bahia & BA & Núcleo de Instrumentos e Medidas - NIM \\
& Universidade Federal de Pernambuco & PE & Núcleo de Estudos em Avaliação Psicológica - NEAP \\
\hline Norte & Universidade Federal do Amazonas & AM & Laboratório de Avaliação Psicológica do Amazonas - LAP-AM \\
& Faculdade Martha Falcão* & AM & Laboratório de Medidas e Avaliação Psicológica - LAMAP \\
\hline
\end{tabular}

*Instituições particulares.

Fonte: Dados recuperados no catálogo de laboratórios do IBAP

Na lista apresentada pelo IBAP (Tabela 2), observa-se que enquanto alguns estados não estão representados (como Goiás, Mato Grosso e Mato Grosso do Sul na região Centro-Oeste; Maranhão, Sergipe e Rio Grande do Norte na região Nordeste; Acre, Amapá, Rondônia, Roraima, Pará e Tocantins na região Norte) há outros, como Distrito Federal, Ceará, Paraíba, Piauí e Amazonas que possuem dois laboratórios cadastrados. Destaca-se que os laboratórios de avaliação psicológica foram mais frequentes nas instituições de ensino públicas, na medida em que apenas a Universidade do Piauí e a Faculdade Martha Falcão são da rede privada.

\section{Discussão}

No presente estudo, realizou-se uma caracterização dos dados de distribuição do ensino de Avaliação Psicológica no âmbito da Pós-graduação Stricto Sensu nas regiões Centro-Oeste, Nordeste e Norte do Brasil. Destaca-se que a última avaliação dos Programas de Pós-graduação Stricto Sensu da CAPES aconteceu em 2017 e não foram encontrados na literatura científica estudos com o intuito de caracterizar a Pós-graduação Stricto Sensu em Psicologia com ênfase em Avaliação Psicológica. 
A realização de novas pesquisas com a Pós-graduação Stricto Sensu, enquanto objeto de investigação, têm sido incentivadas (Féres-Carneiro et al., 2010; Tourinho \& Bastos, 2010) e são importantes para compreender o panorama e desenvolvimento da área.

Os resultados foram apresentados com base em quatro dados principais, a saber: (a)número de pessoas com doutorado e mestrado; (b)quantitativo de pessoas com doutorado e mestrado em Psicologia; (c)número de instituições com Programas de Pós-graduação Stricto Sensu em Psicologia que tenham linha de pesquisa em Avaliação Psicológica; e (d)a quantidade de laboratórios em Avaliação Psicológica. Nessa sequência serão discutidos os dados com o foco na distribuição geográfica para compreender a situação das regiões Centro-Oeste, Nordeste e Norte.

Em relação à quantidade de pessoas com doutorado e mestrado no Brasil, pôde-se perceber a partir dos dados da Figura 1 uma prevalência de mestres. Essa informação faz sentido, haja vista que ao longo dos anos no Brasil existem mais discentes no mestrado, bem como maior quantidade de cursos de mestrado do que de doutorado (CGEE, 2016; Cirani et al., 2015; CAPES, 2010, 2017; INEP, 2016). Notou-se ainda que o Sudeste é a região com maior número absoluto de pessoas com título de doutorado e mestrado. De maneira complementar, a Figura 2 evidenciou a quantidade de pós-graduados por 100 mil habitantes considerando todos os estados brasileiros. Os dados corroboraram a prevalência do Sudeste e do Sul sendo representado por praticamente todos os estados, mas percebeu-se que o Distrito Federal é um estado que impulsiona a região Centro-Oeste e os estados da Paraíba e Rio Grande do Norte fortalecem o Nordeste em termos de quantidade de pós-graduados titulados.

Ao analisar a distribuição de pós-graduados em Psicologia por região, notou-se, de acordo com a Figura 3 que, considerando os números por 100 mil habitantes, a região Sul vem a frente destes percentuais em primeira posição. Posteriormente, a região CentroOeste apresenta um número maior de doutores e mestres em comparação à região Sudeste. Em penúltima posição está a região Nordeste e, em último, a Norte.

Utilizando os dados da Figura 1, que informam a quantidade de concessão de títulos de doutores e mestres por região, esse número está consonante com a predominância de Programas de Pós-graduação Stricto Sensu no eixo Sul-Sudeste (Bastos et al., 2015; Cirani et al., 2015; CAPES, 2017; Féres-Carneiro et al., 2010; Tourinho \& Bastos, 2010). Entretanto, quando foi analisado a quantidade de pós-graduados em Psicologia por
100 mil habitantes cadastrados na Plataforma Lattes, observou-se que a região Centro-Oeste superou os dados do Sudeste (Figura 3). Observa-se que o cadastro na plataforma Lattes é voluntário e, em geral, está relacionada à atuação profissional em Instituições de Ensino Superior e não ao mercado de trabalho em geral. Essa prevalência da região Centro-Oeste frente ao Sudeste pode ter relação com o fato de o Sudeste conseguir absorver os profissionais pós-graduados em indústrias e serviços, por exemplo. Assim, os profissionais dessa região não têm a necessidade de preencher dados na Plataforma Lattes, que está bastante vinculada com o trabalho no campo acadêmico. Essa situação não tende a acontecer da mesma maneira no Centro-Oeste que não possui essa capacidade elevada de agregar os pós-graduados em outros campos de trabalho. Por conseguinte, uma das poucas possibilidades de ingressar no mercado de trabalho é no ensino superior e por isso uma maior adesão ao cadastro na Plataforma Lattes.

Com os dados da Tabela 1, observou-se que não existem instituições com Programas de Pós-graduação Stricto Sensu com concentração em Avaliação Psicológica nas regiões Centro-Oeste, Nordeste e Norte. Mesmo com essa ausência, foram encontradas instituições que possuíam linha de pesquisa na área e com grupos de pesquisa registrados no Diretório de Grupo de Pesquisa do CNPq. O primeiro e ainda único programa com essa área de concentração no Brasil é o da Universidade São Francisco, uma instituição particular, que fica na região Sudeste (Gouveia, 2018; Primi, 2010).

É notório no perfil da Pós-graduação Stricto Sensu em Psicologia, conforme Tabela 1, o predomínio de linhas de pesquisa em Avaliação Psicológica em contraposição a Programas de Pós-graduação Stricto Sensu específicos. Entre as diversas linhas de pesquisa em Psicologia, as que se referem à Avaliação Psicológica não são as mais recorrentes, mas tem se observado um aumento em consonância com o crescimento do número de Programas de Pós-graduação Stricto Sensu (Bastos et al., 2015; Féres-Carneiro et al., 2010; Tourinho \& Bastos, 2010). Na década de 2000, existiam nove instituições com linhas de pesquisa em Avaliação Psicológica nos Programas de Pós-graduação Stricto Sensu em Psicologia, das quais apenas uma delas era na região Centro-Oeste e as regiões Nordeste e Norte não possuíam (Primi, 2010). $\mathrm{O}$ crescimento nas linhas de pesquisa é percebido, pois atualmente há duas no Centro-Oeste, uma no Nordeste e uma no Norte.

Notou-se ainda que das 72 instituições com Programas de Pós-graduação Stricto Sensu em Psicologia, 17 estavam na região Nordeste, nove no Centro-Oeste e 
três no Norte e juntas representaram 39\% dos programas em Psicologia do Brasil. De acordo com Tourinho e Bastos (2010), em 2009 estavam concentrados no eixo sul e sudeste aproximadamente $59 \%$ e $52 \%$, respectivamente, dos mestrados e doutorados em Psicologia. Ainda que tenha se constatado uma distribuição dos programas nas regiões Centro-Oeste, Nordeste e Norte, essa assimetria é observada ao longo dos anos (Bastos et al., 2015; Féres-Carneiro et al., 2010; Tourinho \& Bastos, 2010). Percebeu-se que do total de IES das três regiões, 13 instituições foram responsáveis por 20 programas com mestrado e doutorado, dos quais o Nordeste contou com oito instituições e 11 programas, o Centro-Oeste com quatro instituições e sete programas e o Norte com uma instituição e dois programas. Esses dados evidenciam que teve um aumento de dois programas em relação à avaliação da CAPES de 2013 (Bastos et al., 2015), sendo os dois programas iniciados na região Nordeste.

Destaca-se que todos os estados das regiões Centro-Oeste e Nordeste possuíam instituições com Programa de Pós-graduação Stricto Sensu em Psicologia, enquanto quatro estados da região Norte não possuíam programas, a saber, Acre (AC), Amapá (AP), Roraima (RR) e Tocantins (TO). Em 2013, além desses estados, Mato Grosso e Piauí não tinham programas em Psicologia (Bastos et al., 2015). Esse dado revela que em aproximadamente cinco anos mais dois estados conseguiram ter Programa de Pós-graduação Stricto Sensu em Psicologia.

Na Tabela 1, pôde ser percebido que a maioria das instituições são públicas (69\%), o que revelou uma manutenção do perfil da Pós-graduação Stricto Sensu ser ofertada majoritariamente pela rede pública (FéresCarneiro et al., 2010). Entretanto, das quatro instituições que possuíam linha de pesquisa em Avaliação Psicológica, apenas uma era pública. Essa característica é algo que pode dificultar o acesso dos alunos ao estudo dessa área específica, devido aos custos de mensalidade e incerteza de bolsas de estudo, por exemplo.

Em relação às notas do curso, notou-se uma variação de avaliações de conceito três a seis, mas com predomínio de conceito quatro e três, o que tem sido o mais recorrente na avaliação dos Programas de Pós-graduação Stricto Sensu em Psicologia (Bastos et al., 2015; Tourinho \& Bastos, 2010). Os Programas de Pós-graduação Stricto Sensu em Psicologia com linha de pesquisa em Avaliação Psicológica tiveram conceito três e quatro.

No que se refere aos laboratórios de pesquisa em Avaliação Psicológica, verificou-se o total de 45. Destaca-se que a quantidade de laboratórios de pesquisa no Brasil tem aumentado, pois em 1997, ano de criação do IBAP, existiam menos de 10 (IBAP, 2015). Ao observar a distribuição entre regiões, a soma das regiões Centro-Oeste, Nordeste e Norte não representa metade dos laboratórios da área no Brasil. De maneira detalhada foi possível verificar na Tabela 2 a distribuição deles nessas regiões. Percebeu-se que entre as três regiões houve um predomínio de laboratórios de pesquisa no Nordeste com representação de seis estados. Nas regiões Centro-Oeste e Norte apenas um estado de cada possuía laboratório, a saber, Distrito Federal e Amazonas, respectivamente. Notou-se também que a maioria está em instituições públicas, o que pode revelar maior incentivo das instituições públicas para a organização de laboratórios no intuito de realização de pesquisas.

Esse dado evidencia que, mesmo com o crescimento que é importante para a área de pesquisa, existe uma concentração de laboratórios de pesquisa no eixo Sul-Sudeste. A informação faz sentido tendo em vista que historicamente essas duas regiões têm apresentado a maior quantidade de Programas de Pós-graduação Stricto Sensu e pós-graduados (Bastos et al., 2015; Féres-Carneiro et al., 2010; Tourinho \& Bastos, 2010).

Considera-se importante que instituições e associações, como o IBAP e a ANPEPP, continuem colocando em prática as suas atividades para estabelecerem parcerias, intercâmbio e cooperação entre pesquisadores e centros de pesquisa. Essas ações possibilitam fortalecer os laboratórios existentes e potencializam o surgimento de outros em regiões que carecem, bem como fortalece a Pós-graduação Stricto Sensu em Psicologia (ANPEPP, 2019; Gomes \& Fradkin, 2015; Guzzo et al., 2015; IBAP, 2015).

Notou-se com as informações apresentadas que a Avaliação Psicológica na Pós-graduação Stricto Sensu em Psicologia, principalmente nas regiões Centro-Oeste, Nordeste e Norte, não possui uma ampla rede de ensino. O crescimento foi observado, mas parece que ainda não o suficiente para diminuir as assimetrias entre as regiões (Bastos et al., 2015; Féres-Carneiro et al., 2010; INEP, 2016; Tourinho \& Bastos, 2010). Salienta-se que pela característica técnica que a Avaliação Psicológica possui e pela peculiaridade de desenvolvimento de instrumentos (Bardagi et al., 2015; Borsa, 2016), essa assimetria regional dos programas dificulta o desenvolvimento de estudos que são basais para área. Ao se considerar, por exemplo, a demanda constante de estudos de normatização, bem como de evidências de validade dos instrumentos psicológicos, faz-se necessário considerar as características das diferentes regiões, aspectos culturais, a população de cada contexto, entre

Estudos de Psicologia, 24(2), abril a junho de 2019, 181-192 
outros aspectos. Esses cuidados nas pesquisas são importantes pelo fato de que podem causar impactos e não serem justos ao se usar um instrumento psicológico que não considere as peculiaridades culturais. Logo, as assimetrias nos Programas de Pós-graduação Stricto Sensu em Psicologia e, mais especificamente em Avaliação Psicológica, tendem a dificultar a realização de pesquisas nessas três regiões brasileiras.

É preciso compreender que a qualidade da Pós-graduação Stricto Sensu influencia na formação da graduação, pois o professor com uma formação de qualidade tende a evitar práticas inadequadas no ensino de Avaliação Psicológica (Ambiel et al., 2019; Bardagi et al., 2015; Borsa, 2016; Freires et al., 2017; Noronha, 2003). Assim, é necessário que a graduação e a Pós-graduação Stricto Sensu estejam cada vez mais relacionadas para que possam se fortalecer e contribuir com a formação inicial e continuada do profissional (Menandro et al., 2013; Yamamoto, 2006).

\section{Considerações finais}

A Pós-graduação Stricto Sensu em Avaliação Psicológica se desenvolveu e acompanhou o crescimento nacional, mas ainda assim não indica que esse processo aconteceu de maneira homogênea. O que se percebeu foi que desde o seu surgimento a Pós-graduação Stricto Sensu tem mantido assimetrias entre as cinco regiões brasileiras. O presente trabalho propôs uma caracterização recente sobre a distribuição do ensino de Avaliação Psicológica no âmbito da Pós-graduação Stricto Sensu nas regiões Centro-Oeste, Nordeste e Norte do Brasil e acredita-se que foi possível realizar tal objetivo.

O panorama foi apresentado e os desafios para diminuir as assimetrias regionais aparentam se perpetuar, mesmo com as políticas públicas de indução. Esse perfil é difícil de ser modificado, mas não impossível. O crescimento das regiões Centro-Oeste, Nordeste e Norte, de acordo com dados da CAPES, do INEP, CGEE e de artigos publicados na literatura científica, indicam a possibilidade de mudança e de se ter esperanças de reduzir cada vez mais as assimetrias regionais. Para essa transformação continuar acontecendo é preciso ser levado em conta as raízes históricas, sociais e econômicas desse país de dimensão continental. Unir forças e manter o diálogo entre instituições, associações e governos indica ser o caminho mais breve para a mudança. Ademais, continuar investindo em promoção de políticas públicas para o fortalecimento da Pós-graduação Stricto Sensu é cada vez mais imprescindível.

No presente estudo não foi possível fazer a análise do tema com todos os dados de 2019 , no qual em diversos momentos foram usados dados de 2014. Essa diferença de tempo é decorrente da falta de publicações mais recentes sobre a pós-graduação. Desta feita, salienta-se a importância de realização de pesquisas semelhantes com ênfase em diversas perspectivas, centros de pesquisa, demais áreas de ensino, nível de produção acadêmica, avaliações dos programas, internacionalização, entre tantas possibilidades. Neste trabalho o destaque foi dado às assimetrias regionais, mas sabe-se que essas não são as únicas a serem pesquisadas e enfrentadas. As assimetrias nas mesorregiões e nas áreas de conhecimento também precisam ser revisitadas constantemente em busca de um desenvolvimento mais homogêneo dos Programas de Pós-graduação Stricto Sensu no Brasil.

\section{Referências}

Ambiel, R. A. M., Zuanazzi, A. C., Sette, C. P., Costa, A. R. L., \& Cunha, F. A. (2019). Análise de ementas de disciplinas de avaliação psicológica: novos tempos, velhas questões. Avaliação Psicológica, 18(1), 21-30. doi: 10.15689/ap.2019.1801.15229.03

Associação Nacional de Pesquisa e Pós-Graduação em Psicologia ANPEPP. (2019). Apresentação. Recuperado de https://www.anpepp. org.br/conteudo/view?ID_CONTEUDO=358

Bardagi, M. P., Teixeira, M. A. P., Segabinazi, J. D., Schelini, P. W., \& Nascimento, E. (2015). Ensino da Avaliação Psicológica no Brasil: levantamento com docentes de diferentes regiões. Avaliação Psicológica, 14(2), 253-260. Recuperado de http://pepsic.bvsalud. org/scielo.php?script=sci_arttext\&pid=S1677-04712015000200011

Bastos, A. V. B., Tomanari, G. Y., Trindade, Z. A., \& Andery, M. A. P. A. (2015). The Psychology postgraduate system in Brazil: Current characteristics and challenges for the area. Psicologia: Reflexão e Crítica, 28(Suppl. 1), 23-33. doi: 10.1590/1678-7153.2015284005

Borsa, J. C. (2016). Considerações sobre a formação e a prática em Avaliação Psicológica no Brasil. Temas em Psicologia, 24(1), 131-143. doi: 10.9788/TP2016.1-09

Cirani, C. B. S., Campanario, M. A., \& Silva, H. H. M. (2015). A evolução do ensino da pós-graduação senso estrito no Brasil: análise exploratória e proposições para pesquisa. Avaliação: Revista da Avaliação da Educação Superior, 20(1), 163-187. doi: 10.590/ S1414-40772015000500011

Conselho Nacional de Desenvolvimento Científico e Tecnológico CNPq. (2019a). Glossário grupo de pesquisa. Recuperado de http://lattes.cnpq.br/web/dgp/glossario?p_p_id=54_INSTANCE_ QoMcDQ9EVoSc\&_54_INSTANCE_QoMcDQ9EVoSc_ struts_action=\%2Fwiki_display\%2Fview\&_54_INSTANCE_ QoMcDQ9EVoSc_nodeName=Main\&_54_INSTANCE_ QoMcDQ9EVoSc_title=Grupo+de+pesquisa

Conselho Nacional de Desenvolvimento Científico e Tecnológico CNPq. (2019b). Glossário linha de pesquisa. Recuperado de http://lattes.cnpq.br/web/dgp/glossario?p_p_id=54_INSTANCE_ QoMcDQ9EVoSc\&_54_INSTANCE_QoMcDQ9EVoSc_ struts_action=\%2Fwiki_display\%2Fview\&_54_INSTANCE_ 
Avaliação Psicológica na pós-graduação: um panorama das regiões Centro-Oeste, Nordeste e Norte do Brasil

QoMcDQ9EVoSc_nodeName=Main\&_54_INSTANCE_ QoMcDQ9EVoSc_title=Linha+de+pesquisa

Coordenação de Aperfeiçoamento de Pessoal de Nível Superior - CAPES. (2010). Plano Nacional de Pós-Graduação-PNPG 2011-2020 (Vol.1). Brasília, DF: Autor.

Coordenação de Aperfeiçoamento de Pessoal de Nível Superior CAPES. (2017). Relatório Final 2016/2017 - Comissão Especial de Acompanhamento do PNPG 2011-2020. Recuperado de https://www. capes.gov.br/images/stories/download/relatorios/231117-RelatorioPNPG-Final-2016-CS.pdf

Coordenação de Aperfeiçoamento de Pessoal de Nível Superior - CAPES. (2019). Sistema de Informações Georreferenciadas. Recuperado de https://geocapes.capes.gov.br/geocapes/

Féres-Carneiro, T., Bastos, A. V., Feitosa, M. A. G., Seidl-de-Moura, M. L., \&Yamamoto, O.H.(2010). Lacunas, metas e condições para a expansão da pós-graduação em psicologia no país. Psicologia: Reflexão e Crítica, 23(Suppl. 1), 11-24. doi: 10.1590/S0102-79722010000400003

Freires, L. A., Silva Filho, J. H., Monteiro, R. P., Loureto, G. D. L., \& Gouveia, V. V. (2017). Ensino da avaliação psicológica no Norte brasileiro: analisando as ementas das disciplinas. Avaliação Psicológica, 16(2), 205-214. doi: 10.15689/AP.2017.1602.11

Gomes, W. B., \& Fradkin, C. (2015). Historical notes on Psychology in Brazil: The creation, growth and sustenance of postgraduate education. Psicologia: Reflexão e Crítica, 28(Suppl. 1), 2-13. doi: 10.1590/1678-7153.2015284002

Gouveia, V. V. (2018). Formação em Avaliação Psicológica: situação, desafios e diretrizes. Psicologia: Ciência e Profissão, 38(spe), 74-86. doi: 10.1590/1982-3703000208641

Guzzo, R. S. L., Linhares, M. B. M., Teodoro, M. L. M., \& Koller, S. H. (2015). Perspectives and challenges regarding brazilian policies for research and postgraduate studies in Psychology. Psicologia: Reflexão e Crítica, 28(Suppl. 1), 34-39. doi: 10.1590/1678-7153.2015284006

Hutz, C. S., Rocha, M. L., Spink, M. J. P., \& Menandro, P. R. M. (2010) Perfil, avaliação e metas de produção intelectual dos programas de pós-graduação em Psicologia. Psicologia: Reflexão e Crítica, 23(Suppl. 1), 25-34. doi: 10.1590/S0102-79722010000400004
Instituto Brasileiro de Avaliação Psicológica - IBAP. (2015). Catálogo dos laboratórios de Avaliação Psicológica no Brasil. Recuperado de http:// www.ibapnet.org.br/congresso2015/arquivos/CATALOGO_DOS LABORATORIOS_DE_AVALIACAO_PSICOLOGICA_NO_BRASIL.pdf

Instituto Nacional de Estudos e Pesquisas Educacionais Anísio Teixeira INEP. (2016). Relatório do $1^{\circ}$ ciclo de monitoramento das metas do PNE: biênio 2014-2016. Recuperado de http://download.inep.gov.br/ outras_acoes/estudos_pne/2016/relatorio_pne_2014_a_2016.pdf

Menandro, P. R. M., Tourinho, E. Z., Bastos, A. V. B., \& Yamamoto, O. H. (2013). Graduate and undergraduate studies: Neighbors without affinity? Paidéia, 23(55), 187-196. doi:10.1590/1982-43272355201306

Centro de Gestão e Estudos Estratégicos - CGEE. (2016). Mestres e doutores 2015 - Estudos da demografia da base técnico-científica brasileira. Brasília, DF: Autor. Recuperado de https://www.cgee.org.br/ documents/10182/734063/Mestres_Doutores_2015_Vs3.pdf

Noronha, A.P.P.(2003). Docentes dePsicologia: formação profissional.Estudos de Psicologia, 8(1), 169-173. doi: 10.1590/S1413-294X2003000100019

Nunes, M.F.O., Muniz, M., Reppold, C.T., Faiad, C., Bueno, J.M.H., \& Noronha, A.P.P.(2012). Diretrizes para o ensino de avaliação psicológica. Avaliação Psicológica, 11(2), 309-316. Recuperado de http://pepsic.bvsalud.org/ scielo.php?script=sci_arttext\&pid=S1677-04712012000200016\&lng= pt\&tlng $=\mathrm{pt}$

Primi, R. (2010). Avaliação Psicológica no Brasil: fundamentos, situação atual e direções para o futuro. Psicologia: Teoria e Pesquisa, Brasília, 26(spe), 25-35. doi: 10.1590/S0102-37722010000500003

Tourinho, E. Z., \& Bastos, A. V. B. (2010). Desafios da pós-graduação em Psicologia no Brasil. Psicologia: Reflexão e Crítica, 23(Suppl. 1), 35-46. doi: 10.1590/S0102-79722010000400005

Yamamoto, O. H. (2006). Graduação e pós-graduação em Psicologia: relações possíveis. Revista Brasileira de Pós-Graduação, 3(6), 270-281. doi: 10.21713/2358-2332.2006.v3.110

Yamamoto, O., \& Menandro, P. (2004). A avaliação dos programas de pós-graduação em Psicologia. Temas em Psicologia, 12(1), 82-91. Recuperado de http://pepsic.bvsalud.org/scielo. php?script=sci_arttext\&pid=S1413-389X2004000100009

Gabriel Vitor Acioly Gomes, Mestre em Educação pela Universidade Estadual do Ceará (UECE), é doutorando no Programa de

Pós-graduação em Psicologia da Universidade São Francisco - USF. Endereço para correspondência: Av. Dr. Silas Munguba, 1700, Campus do Itaperi. Fortaleza-CE, Brasil. CEP: 60.714-903. Telefone: (85) 3101-9987. Email: gabrigom93@hotmail.com

Camila Costa e Silva, Graduada em Psicologia pela Pontifícia Universidade Católica de Goiás - PUC/Goiás, mestranda na Pontifícia Universidade Católica de Goiás -PUC/Goiás. Email: camilacosta1802@gmail.com

Daniela Sacramento Zanini, Doutora em Psicologia Clínica e da Saúde pela Universidad de Barcelona - UB, Espanha, Pós-doutora pela Universidad de Barcelona - UB, Espanha, é Professora Adjunta na Pontifícia Universidade Católica de Goiás - PUC/Goiás. Email: dazanini@yahoo.com

Lucila Moraes Cardoso, Doutora em Psicologia pela Universidade São Francisco - USF, é Professora Adjunta na Universidade Estadual do Ceará - UECE. Email: lucila.cardoso@uece.br 